\title{
El Colegio Nacional de Educación a Distancia: una innovación en la educación de adultos
}

\author{
Juan Martín Rojas Gómez
}

Docente, Escuela de Educación, Universidad Estatal a Distancia(UNED), San José, Costa Rica

Recibido: 22 de abril de 2010

\section{RESUMEN}

Se destaca la labor que realiza el Colegio Nacional de Educación a Distancia (CONED), la cual destaca por ser una innovación en el campo de la Educación Media en Costa Rica y un aporte de la Universidad Estatal a Distancia (UNED) y el Ministerio de Educación Pública (MEP) a favor de las personas mayores de dieciocho años que, por diversas razones, no han concluido su educación secundaria. La propuesta del CONED rescata la importancia de la educación para adultos, un derecho fundamental y un elemento vital para el desarrollo personal y social. Se presenta el plan de estudios y los aspectos relacionados con la matrícula y sedes con que cuenta el CONED, como un paso firme para lograr la universalización de la Educación Media. Finalmente, se relaciona el surgimiento de este Colegio como un aporte y una demostración de responsabilidad social de la UNED, institución líder en los procesos de educación a distancia, a favor de los miembros de las clases sociales menos favorecidas.

\section{PALABRAS CLAVE}

Educación media, universalización de la educación secundaria.
Aceptado: 16 de setiembre de 2010
This article have to highlight the work of the Colegio Nacional de Educación a Distancia (CONED) is presented as an innovation in the field of Media Education in Costa Rica and a contribution from the Universidad Estatal a Distancia (UNED) and Ministerio de Educación Pública (MEP) for people over eighteen years, for various reasons, have not completed high school. CONED the proposal preserves the importance of adult education as a fundamental and vital to personal and social development. We present the curriculum and issues related to enrollment and places available to the CONED, as a firm step towards achieving universal secondary education. Finally, it relates the emergence of this college as the contribution of the UNED, the leading institution in distance education processes and a demonstration of social responsibility, on behalf of members of disadvantaged social classes.

\section{KEY WORDS}

Secondary education, universal secondary education.

\section{INTRODUCCIÓN}

Desde sus inicios como República soberana e independiente, Costa Rica ha considerado la educación como uno de sus pilares más importantes, sobre los que descansa el desarrollo y la movilidad social. Es la savia de la cual se nutren los principios de igualdad y paz social. El Estado, como ente rector de todo el sistema educativo, adoptó acciones para fortalecer su desarrollo y evolución, 
hasta convertir a nuestro país en un ejemplo ante las naciones del mundo: un país pequeño que prefiere invertir en educación, antes que en armas, y cuyo estilo de vida ha propiciado que sus habitantes puedan alcanzar metas y objetivos que les permiten satisfacer sus necesidades personales, familiares y profesionales.

Lamentablemente, no se ha fortalecido la acción de continuidad que requiere el sector educativo, olvidando que lo que no cambia, tampoco mejora. John F. Kennedy, citado por Adair (1990: 24), señala: "Existen riesgos y costos para un programa de acción. Pero están muy lejos de los riesgos y costos a largo plazo de una cómoda inacción", precisamente esto ocurrió en nuestro país, con la inacción comenzaron a llegar los problemas sociales que hoy agobian a nuestra sociedad. El sistema educativo no escapa de ellos; sus problemas se multiplicaron: la calidad de la educación bajó, y como consecuencia se presenta la deserción, entre otras causas por el desfase entre lo aprendido en las aulas y lo vivido cotidianamente, se dio un retraso con respecto del desarrollo académico en otros países.

Sin duda, la incapacidad para retener al alumnado es uno de los principales problemas que por años ha afectado al sistema educativo costarricense. Así lo ha señalado el décimo primer Informe del Estado de la Nación, programa Estado de la Nación en Desarrollo Humano sostenible (2005: 273), el cual indica que "el Sistema Educativo costarricense, según ha señalado desde hace varios años el informe Estado de la Nación, ha demostrado incapacidad para retener a las y los estudiantes" en los centros educativos, hasta concluir los ciclos correspondientes.

Los niños, niñas y jóvenes que abandonan el Sistema Educativo, se han constituido en un sector muy amplio, de acuerdo con las estadísticas del Ministerio de Educación Pública, "se prevé que de cada 1000 (estudiantes) que ingresaron al sistema en el año 2000, se graduarán 349", (Programa Estado de la Nación en Desarrollo Humano sostenible, 2005: 276). Lo anterior permite realizar dos afirmaciones: que este sector aumenta considerablemente cada año, de mantenerse la misma condición y que se hace urgente la atención a esta población, que deserta.

Muchas son las razones por las que los jóvenes abandonan las aulas, el Informe del Estado de la Nación (2005) reconoce factores académicos, además de las motivaciones o presiones económicas y familiares, otros autores como Ander-Egg, van más allá y consideran que "el fracaso escolar, como todos los factores sociales, es una manifestación multicausal en los que casi todos las causas están presentes en cada caso, con incidencia diferente de cada una de ellas pero, todas las circunstancias, retroalimentándose entre sí" (2005: 107), por tanto, plantean una seria problemática que tiene su origen en variados aspectos, el mismo AnderEgg destaca los siguientes:

a) Falta de interés y motivación: Se considera que los estudiantes están obligados a participar con mayor compromiso en las obligaciones que la escuela o colegio demandan, como garantía de éxito.

b) Trastornos personales: Tomando en cuenta todos los trastornos, tanto los físicos, los visuales o los auditivos; como los que se producen por el hambre o una dieta insuficiente, los psicológicos que demandan tratamiento de otros profesionales o los que pueden generar un desarrollo cerebral inadecuado.

c) Causas afectivas: Ambos extremos, la falta de cariño y la sobreprotección pueden derivar en baja autoestima o inseguridad.

d) Causas cognitivas: Producto del desajuste entre la edad cronológica y la intelectual.

e) Bajo valor que otorga la familia a la educación o la desvalorización total: Entre otras causas, depende del nivel educativo de los padres de familia. 
f) Divorcio entre la familia y la escuela: Con frecuencia se otorga a la escuela la responsabilidad total de la educación de los hijos, la participación de la familia es casi nula o se convierte en vigilante del proceso y no en coadyuvante.

g) Medio social y situación económica: Cuando la realidad obliga a satisfacer otras necesidades como el alimento, la salud o el vestido, antes que la educación.

h) Profesores y maestros no suficientemente preparados o motivados: La formación inicial académica no ha sido la mejor o la inestabilidad laboral o salarial genera que el personal docente no se involucre y se comprometa con el proceso educativo.

i) Inadecuados métodos de enseñanza/ aprendizaje: El uso de acciones rutinarias en el desarrollo de las lecciones, provoca que se tornen aburridas y poco atractivas para los estudiantes.

Lo anterior, entre otras razones, produce la desbandada de los estudiantes hacia otras actividades donde se sienten mejor, con los grupos de amigos, con los juegos electrónicos y en el peor de los casos pasar el tiempo en la calle, donde los malos ejemplos, la delincuencia y la corrupción campean.

Actualmente, se debe considerar que en Costa Rica la demanda de educación media para las personas menores de edad es muy amplia: los colegios diurnos y nocturnos no tienen la capacidad de espacio para atender a toda la población que exige este servicio. La situación se agrava cuando se trata de la población adulta; el Ministerio de Educación Pública lleva a cabo una loable labor por medio de su Departamento de Educación de Adultos; no obstante, no cubre las necesidades de la totalidad de la población, por razones lógicas de infraestructura, personal, recursos económicos y material didáctico, entre otras.
Costa Rica, país que aspira a ser "desarrollado", no puede obviar que muchos de sus jóvenes en edad productiva no han logrado concluir su educación media. Además de este aspecto, son muchos los motivos que tiene el Estado para garantizar a la población adulta el derecho a la educación: se trata de situar a la persona en el centro de toda acción, considerar que la educación abre puertas y garantiza una mejor condición personal y comunal de todos los habitantes del país, o como lo establece el Informe del Estado de la Nación (Programa Estado de la Nación en Desarrollo Humano Sostenible 2005: 273) "porque impacta a la sociedad en diversos planos: tiene efectos sociales (propicia la movilidad social, contribuye a disminuir la pobreza y a crear un clima propicio para estimular la formación educativa", por tanto el compromiso debe asumirse con la responsabilidad que el futuro del país demanda.

Otra razón digna de considerar está relacionada con las generaciones futuras. En la figura 1 se expone lo que también señala el Informe del Estado de la Nación (2005: 273), en el sentido de que "existen evidencias de que la escolaridad de los padres incide fuertemente en el nivel educativo de sus hijos".

El análisis de este gráfico permite establecer una relación inversamente proporcional, a menor escolaridad de los padres de familia, mayor posibilidad de que sus hijos no ingresen o concluya los niveles que establece el sistema educativo, en especial el de la educación media, lo que conduce a problemas mayores: dificultades para obtener empleos bien remunerados, bajos ingresos económicos, en pocas palabras, la vía para llegar a la pobreza y consecuentemente la simiente de todos los problemas sociales que genera.

\section{- Fortelecimiento de la educación de adultos}

La educación es un derecho fundamental, así lo dictan la legislación y los derechos huma- 


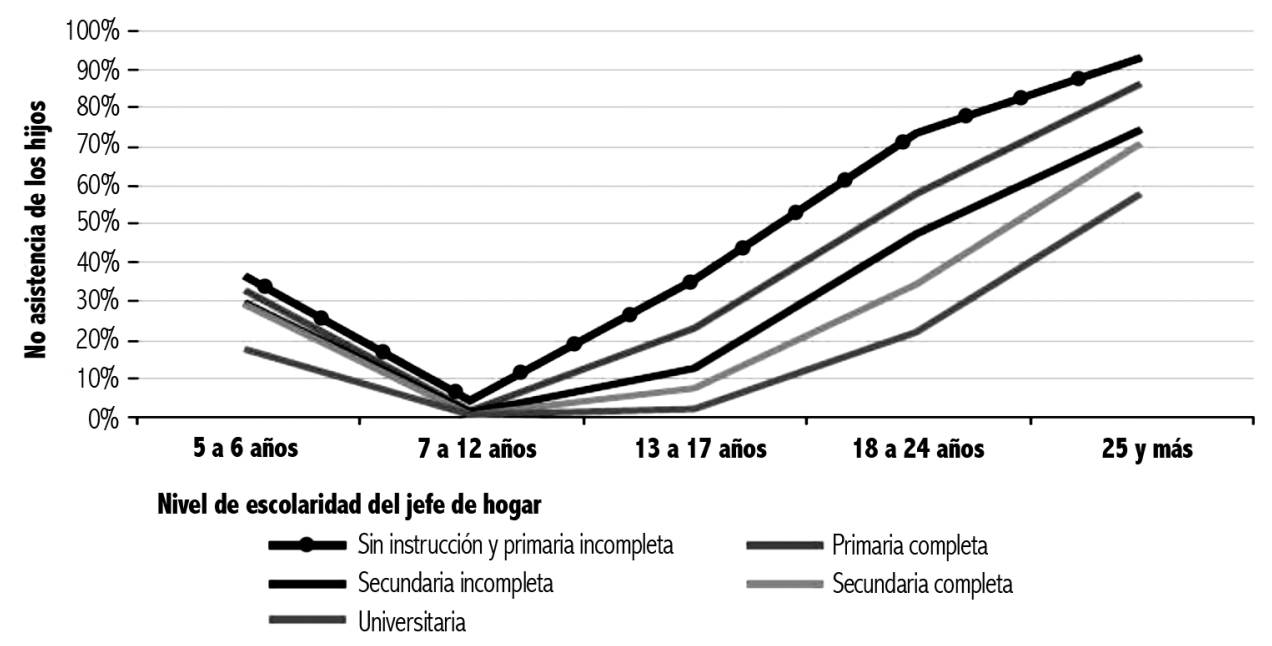

Figura 1. Relación entre la no asistencia a la educación, según educación de los padres.

Fuente: Informe del Estado de la Nación 2005.

nos, pero cabe destacar, como lo establece Gimeno Sacristán (2005: 126) que "...el sujeto del derecho a la educación es el ser humano, no está restringido al niño o los menores. En la práctica, este reconocimiento ampara y apoya directamente la necesidad de la educación de los adultos y el aprendizaje permanente como una ampliación de la cobertura de aquel derecho fundamental".

Como señala Sacristán, la educación de adultos demanda atención, si bien la inversión en educación de niños y jóvenes representa el mayor porcentaje de los recursos destinados a la educación, es imperante que no se descuide este sector, fortaleciendo las iniciativas e innovaciones en este campo. Costa Rica ha comprendido la importancia de la educación de adultos, en la Constitución Política, en el Título VII, artículo 83, establece que "El Estado patrocinará y organizará la educación de adultos, destinada a combatir el analfabetismo y a proporcionar oportunidad cultural a aquéllos que deseen mejorar su condición intelectual, social y económica". Con esa responsabilidad y sobre la base legal, se lograron muchos éxitos en el pasado y se busca mejorar cada día en este campo.

La finalidad de este artículo es destacar la labor que en este sentido ha desarrollado el Colegio Nacional de Educación a Distancia (CONED), la cual surge como una propuesta a favor de la población mayor de dieciocho años que no ha concluido su educación media y desarrollada conjuntamente con el Ministerio de Educación Pública. Esta propuesta surge como innovación en la educación media costarricense por medio de una metodología alternativa: estudiar a distancia.

Aunque existen en el ámbito nacional diversas ofertas para obtener el Tercer Ciclo y la Educación Diversificada, ninguna aplica la metodología de educación a distancia, tal como lo propone el Colegio Nacional de Educación a Distancia (CONED), emulando en su accionar a la Universidad Estatal a Distancia (UNED), en cuanto al modelo de evaluación y el uso del material didáctico.

El Colegio Nacional de Educación a Distancia es una innovación, en cuanto surge como "una forma de mejorar la práctica pedagógica 
desde la reflexión y la propuesta de alternativas diferentes, para así satisfacer las necesidades básicas de aprendizajes significativos, en un contexto cultural e histórico dado" (UNED, 2007), que permite estudiar sin descuidar sus otras obligaciones sociales como trabajar y atender la familia, también puede considerarse como innovación, según lo que propone Adair (1990), "una introducción deliberada y específica de lo que es nuevo, con miras a alcanzar las metas" (p.8), en este caso, la culminación de la educación media. Desde ambas perspectivas, para mejorar la práctica pedagógica o una introducción deliberada, se debe reconocer este esfuerzo, considerando que, mientras se atiende esta necesidad de educación para adultos, el Estado camina por sendas que le permiten mejorar las expectativas de estudio de los niños y niñas en edades muy cortas, y para aquellos que aún no han nacido.

En este punto, es indispensable aclarar que el tema no es de carácter institucionalista o políti$\mathrm{co}$, sino de responsabilidad social y que merece atención para superar todos los obstáculos que impiden el desarrollo integral de los habitantes, por lo tanto, debe ser atendido con prontitud y eficacia, comprendiendo la trascendencia que posee para el desarrollo del país. Así, lo propone Gimeno Sacristán (2005: 117) "reconociendo el papel que desempeña el conocimiento en el desarrollo económico, en la prosperidad cultural y en la satisfacción personal", por tanto, es de vital importancia que surjan instituciones que fortalezcan la universalización de la enseñanza media.

\section{- Un paso al frente en la universalización de la educación media}

El Colegio Nacional de Educación a Distancia (CONED), surge como una propuesta conjunta de la Universidad Estatal a Distancia (UNED) y el Ministerio de Educación Pública (MEP), por medio de un Convenio de Cooperación
Institucional, por acuerdo 02-27-04 del Consejo Superior de Educación, aprobado en sesión 2704, con el cual se dispone la creación del Colegio Nacional de Educación a Distancia, para atender el Tercer Ciclo de la Educación General Básica y la Educación Diversificada.

El plan de estudio propuesto se conforma de seis asignaturas: Español, Matemáticas, Ciencias Naturales- Biología, Educación Cívica, Estudios Sociales e Inglés.

Además se ofrecen cursos para el crecimiento personal, tales como: Inglés Conversacional I y II, Gestión Empresarial, Computación I y II, Contabilidad I y II, Relaciones Públicas y Técnicas de Estudio a Distancia.

El 28 de julio del año 2005 el CONED inicia sus labores, con una población de trescientos tres estudiantes, distribuidos en ocho sedes, ubicadas en lugares donde la UNED cuenta con uncentro universitario: Nicoya, Liberia, Palmares, Heredia, San José, Turrialba, Limón y Ciudad Neily.

Ante la carencia de material didáctico, el CONED ha utilizado las unidades didácticas elaboradas por el Instituto Costarricense de Enseñanza Radiofónica (ICER). En la actualidad se cuenta con una unidad didáctica propia, que es la de Español $7^{\circ}$ y las demás están en proceso de elaboración.

Han pasado cuatro años de su fundación y funcionamiento, el CONED ha crecido, tanto en cantidad de sedes como en población estudiantil, se puede clasificar como uno de los colegios de educación formal más grandes de Costa Rica, en la actualidad su matrícula supera los cinco mil trescientos estudiantes. En la figura 2 se puede observar la ubicación de las sedes del CONED en el territorio nacional.

En este momento, existe la petición por parte de muchas comunidades para la apertura de nuevas sedes del CONED en todo el país; lo que debe ser motivo de análisis para las autoridades de la UNED y el MEP, en especial para atender las zonas costeras y el norte del país, donde 


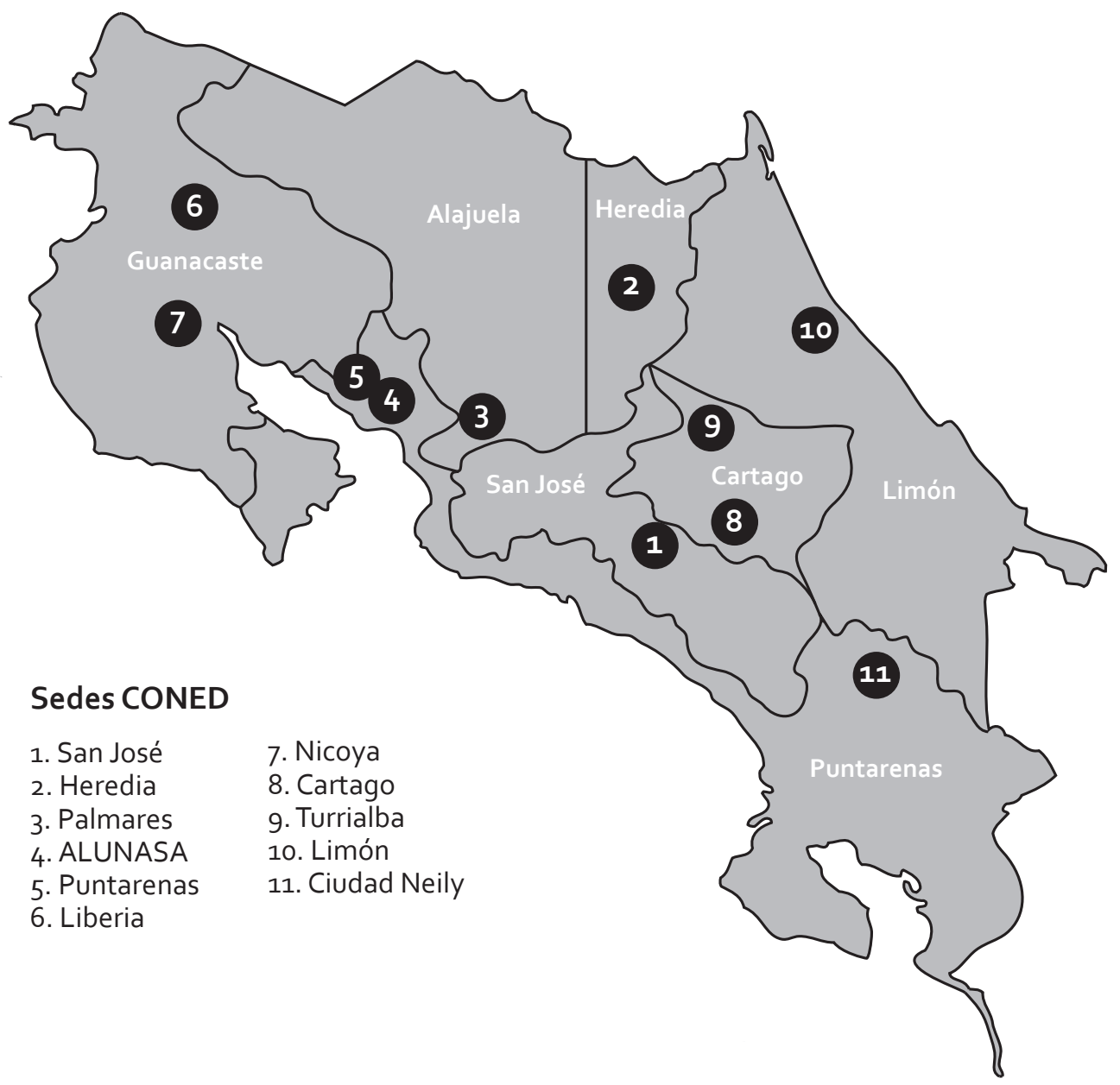

Figura 2. Localización de las sedes del Colegio Nacional de Educación a Distancia.

Fuente: Documentos CONED

los accesos a la educación secundaria pueden ser más difíciles para la población adulta. Así, el Informe del Estado de la Nación establece que "la universalización de la primaria es un hecho en Costa Rica, lograr la universalización de la secundaria es un desafío inaplazable en el actual momento histórico" (Programa Estado de la Nación, 2005: 275).

Sin duda, el Colegio Nacional de Educación a Distancia (CONED), apoyado en la trayectoria de éxito de la UNED en los procesos de educación a distancia, es un cimiento para lograr la universalización de la enseñanza secundaria en Costa Rica. El reto educativo es grande y la inversión que hace el Estado también, sin embargo, para el estudiante no, pues una de las características de la educación a distancia es que abarata los costos pues no requiere desplazarse grandes distancias para llegar al centro educativo; por tanto, no debe invertir en alimentación o transporte, ni en material didáctico adicional.

Por otra parte, la participación e inserción de la mujer en este proceso es también un aspecto positivo; las estudiantes del CONED son jefes de hogar, casadas, mayores o jóvenes que en 
algún momento vieron limitado su esfuerzo y ahora visualizan esta opción de educación a distancia como una forma de retomar sus metas personales y profesionales, lo que se muestra en el cuadro 1, el cual resume la matrícula histórica del CONED.

Los datos estadísticos del CONED reflejan aspectos muy importantes, por ejemplo, para el año 2009 la población estudiantil se conformaba de un total de tres mil ochenta y tres (3083) mujeres, mientras que los varones sumaban dos mil doscientos cincuenta y cuatro (2254), lo que representa que $58 \%$ de los estudiantes son mujeres.

Este aspecto es motivo de orgullo para el CONED, por la importancia que reviste para el futuro de Costa Rica, como se señaló los hijos de padres con elevados niveles académicos tienen mayores posibilidades de éxito en el sistema educativo, de esta manera se garantiza un futuro mejor a las nuevas generaciones.

El CONED recién inicia sus labores. Su trabajo es arduo y su aporte al desarrollo del país re- sulta vital, sobre todo en la lucha por la universalización de la educación secundaria. Debe ser fortalecido y mejorar su accionar, necesita del apoyo de la Universidad Estatal a Distancia y del Ministerio de Educación Pública, pero sobre todo del cuerpo docente, así lo confirman Sancho, Hernández, Carbonell, Tort, Simó y Sánchez (1998: 39) al "considerar el importante papel que tiene el profesorado en una innovación, si se pretende favorecer algún tipo de cambio", en el caso del CONED, el desarrollo de una metodología de educación a distancia en adultos que cursan la educación media.

\section{¿Por qué la universidad estatal a distancia?}

La respuesta a esta interrogante es porque la UNED ha demostrado su liderazgo por más de treinta años en el campo de la educación a distancia, porque es fiel a sus raíces, lucha por los grupos sociales menos favorecidos y desde su modelo pedagógico establece Lineamientos

Cuadro 1. Matrícula histórica del CONED.

\begin{tabular}{|c|c|c|c|c|c|c|c|c|c|c|c|c|c|c|c|}
\hline \multirow[b]{2}{*}{ Sede } & \multicolumn{3}{|c|}{2005} & \multicolumn{3}{|c|}{2006} & \multicolumn{3}{|c|}{2007} & \multicolumn{3}{|c|}{2008} & \multicolumn{3}{|c|}{2009} \\
\hline & M & $\mathrm{H}$ & $\mathrm{T}$ & M & H & $\mathrm{T}$ & M & H & $T$ & M & H & $\mathrm{T}$ & M & H & $\mathbf{T}$ \\
\hline San José & 416 & 287 & 703 & 571 & 535 & 1106 & 667 & 538 & 1205 & 784 & 699 & 1483 & 835 & 727 & 1562 \\
\hline Palmares & & & & 133 & 120 & 253 & 141 & 122 & 263 & 171 & 132 & 303 & 227 & 174 & 401 \\
\hline Nicoya & & & & 46 & 25 & 71 & 89 & 50 & 139 & 157 & 114 & 271 & 132 & 88 & 220 \\
\hline Ciudad Neily & & & & 155 & 80 & 235 & 205 & 132 & 337 & 273 & 153 & 426 & 256 & 122 & 378 \\
\hline Limón & & & & 75 & 37 & 112 & 193 & 80 & 273 & 362 & 197 & 559 & 391 & 191 & 582 \\
\hline Liberia & & & & 175 & 84 & 259 & 182 & 71 & 253 & 209 & 81 & 290 & 225 & 80 & 305 \\
\hline Turrialba & & & & 96 & 46 & 142 & 154 & 77 & 231 & 193 & 116 & 309 & 224 & 157 & 381 \\
\hline Heredia & & & & 158 & 125 & 283 & 264 & 242 & 506 & 327 & 304 & 631 & 364 & 335 & 699 \\
\hline ALUNASA & & & & 37 & 48 & 85 & 84 & 80 & 164 & 108 & 84 & 192 & 136 & 116 & 252 \\
\hline Cartago & & & & & & & & & & 144 & 156 & 300 & 229 & 201 & 430 \\
\hline Sarapiquí & & & & & & & & & & 8 & 1 & 9 & 21 & 12 & 33 \\
\hline Puntarenas & & & & & & & & & & & & 0 & 43 & 51 & 94 \\
\hline TOTAL & & 703 & & & 2546 & & & 3371 & & & 4773 & & & 5337 & \\
\hline
\end{tabular}

Fuente: Documentos CONED. 
de Políticas Institucionales, los cuales favorecen el desarrollo de las personas, entre otros aspectos, los siguientes:

» La autonomía y el aprender a aprender.

"Las competencias intelectuales, sociales, personales y profesionales.

" Las habilidades para encontrar, seleccionar y transformar información en conocimiento relevante para la formación personal y la transformación social.

Este espíritu institucional también lo imprime al CONED y con ello traslada su experiencia al campo de la educación media. En su decisión de crear y atender este colegio, la Universidad Estatal a Distancia cumple con uno de sus objetivos establecidos en el Modelo Pedagógico (2007: 6) "e) Proporcionar instrumentos adecuados para el perfeccionamiento y formación permanente de todos los habitantes", a la vez que evidencia los cinco aspectos propios de un líder creativo, que señala Adair (1990: 88-90) y se destacan a continuación:

»Una voluntad para aceptar el riesgo. La UNED asume el reto de establecer un colegio, destinando parte de su presupuesto a la dotación de recursos para su funcionamiento y desarrollo, pese a las limitaciones que enfrenta.

Una habilidad para trabajar con ideas a medio hornear. La idea de un colegio a distancia, es algo nuevo, su desarrollo requiere de toda su experiencia y la garantía de éxito va a depender de la conjugación de múltiples factores, entre los que se destaca la voluntad política y el compromiso de los actores. Una voluntad para flexibilizar las reglas. Se demuestra la voluntad de la UNED por lograr que el CONED tenga éxito, primero al destinar fondos de la educación superior a la educación media, también al crear material para este nivel y a la vez lidiar con toda una nueva experiencia de enseñanza.

» Habilidad para responder rápidamente. Por su espíritu de entrega y compromiso a favor de las clases sociales menos privilegiadas, la UNED buscó una respuesta a la problemática de miles de costarricenses mayores de edad, quienes no han concluido su educación media, ofrece una opción que les permite desarrollarse personal y profesionalmente, con el propósito de obtener mejores empleos y por ende una mejor calidad de vida.

»Entusiasmo personal. Las autoridades del la Universidad Estatal a Distancia, desde la Rectoría, Vicerrectoría de Planificación, el Consejo Universitario y otras dependencias, se han identificado con el CONED, cada cual en su campo ha evidenciado su compromiso y ha realizado su aporte para la lograr que este proyecto sea exitoso.

Por las razones expuestas, la creación del Colegio Nacional de Educación a Distancia no podría haberse gestado en ninguna otra institución de este país; por sus características, su población meta, su compromiso con el desarrollo personal y social, solo se pudo haber concebido en la Universidad Estatal a Distancia, cuyas autoridades han leído el contexto histórico y actual de Costa Rica y con valentía han levantado el estandarte a favor de quienes menos tienen y han abierto una posibilidad para mejorar su condición personal, a la vez que contribuyen con la construcción de un mejor país.

\section{REFERENCIAS}

Adair, J. (1990). El reto gerencial de la innovación. (Trad. J. Villamizar) Bogotá, Colombia: The talbot. (Original en inglés, 1190). 
Ander-Egg, E. (2005). Debates y propuestas sobre la problemática educativa: algunas reflexiones sobre los retos del futuro inmediato. Argentina: Homo Sapiens Ediciones.

Colegio Nacional de Educación a Distancia. (2009). Informe de labores 2005-2009. San José, Costa Rica.

Constitución Política de Costa Rica. Consultado el 13 de febrero de 2010, http://www.cesdepu. com/nbdp/copol2.htm

Convenio de Cooperación Interinstitucional para la Apertura y Funcionamiento del Colegio Nacional de Educación a Distancia entre el Ministerio de Educación Pública y la Universidad Estatal a Distancia. 28 de julio de 2005.
Gimeno, J. (2005). La educación que aún es posible: Ensayos acerca de la cultura para la educación. Madrid: Ediciones Morata, S.L.

Programa Estado de la Nación en Desarrollo Humano Sostenible. Undécimo Informe Estado de la Nación en Desarrollo Humano sostenible. San José: Programa Estado de la Nación, 2005.

Sancho, J.; Hernández, F.; Carbonell, J.; Tort, T.; Simó, N.; Sánchez, E. (1998). Aprendiendo de las innovaciones en los centros. Barcelona: Octaedro.

Universidad Estatal a Distancia. (2007). Modelo pedagógico. San José, Costa Rica: EUNED. 
